ECONOMIC THEMES (2019) 57(4): 415-432

sciendo

DOI 10.2478/ethemes-2019-0024

\title{
COMPETITIVENESS AND THE EU ACCESSION PROCESS: CAN CANDIDATE COUNTRIES BECOME COMPETITIVE AS EU COUNTRIES?
}

\author{
Jelena J. Stanković
}

University of Niš, Faculty of Economics, Republic of Serbia

$\bowtie$ jelena.stankovic@eknfak.ni.ac.rs

Marija Džunić

University of Niš, Faculty of Economics, Republic of Serbia

$\triangle$ marija_dzunic@ni.ac.rs

Vesna Janković Milić

University of Niš, Faculty of Economics, Republic of Serbia

$\bowtie$ vesna.jankovic@eknfak.ni.ac.rs

UDC

339.137 .2

(4-672EU)

Review paper

\begin{abstract}
Economic growth and competitiveness are usually analysed at the level of the national economy in traditional economic research. The problem of competitiveness within this line of thought is mainly regarded from the perspective of determining the sources of sustainable growth, which makes the economy more competitive than others. Competitiveness, therefore, is a multidimensional concept, which includes a range of factors, such as institutions, infrastructure, macroeconomic environment, market, human capital and technological development. Also, the process of joining the European Union significantly stimulates the development of the exact categories that are relevant for acceleration of the economic development. The aim of the paper is to assess the competitiveness of the candidate or potential candidate countries for membership in the European Union, through a comparative analysis of their competitiveness vis-à-vis EU countries. The results indicate that the competitiveness of the EU 15 countries, measured by Global Competitiveness Index and GDP per capita, is statistically significantly higher than the competitiveness of EU country group enlarged in the period 2004-2013, also compared to EU candidate or potential candidate countries. However, when it comes to the pillar of competitiveness related to the macroeconomic environment, according to the latest Global Competitiveness Report (2017-2018), the scores of the EU countries associated with enlargements in the period 2004-2013 are statistically significantly better than the EU 15 countries.
\end{abstract}




\section{Introduction}

Although the concept of competitiveness has initially been associated with enterprises, the vast amount of literature accentuates its multidimensional nature, applying the concept at the level of different territorial units, such as regions, industries and ultimately, national economies. The micro aspects of competitiveness have dominated in the literature, relating the enterprise competitiveness to its market share, profitability or growth, based on which enterprises compete with each other in the market. Therefore, the competitiveness of the enterprise represents its ability to survive and prosper in a competitive environment (Porter, 1990). The competitiveness of individual economic units is related to the achievement of competitive advantages in relation to other enterprises in industry. This means that the enterprise is able to produce goods and services more efficiently than competitors, based on innovations, product diversification and increased productivity.

In recent years, owing to the globalization trends, the concept of national competitiveness has received a lot of attention, associated with the issues of welfare and growth. The main question of these analyses is to explain why some countries gain competitive advantage, develop faster and become richer, while other countries don't. It became obvious that differences in economic performance cannot simply be attributed to the traditional factors, such as industrial policy or technology transfer. Measuring national competitiveness has been a controversial issue from the very beginning, ever since the first composite indices have been constructed for this purpose. As a response to the increasing interest of policy makers, numerous research institutions, analysts and consultants have taken to the task of providing solutions for benchmarking competitive performance of different countries.

Based on the most widely used measure of national competitiveness, the Global Competitiveness Index published by the World Economic Forum, this paper aims to assess the differences in the competitiveness of different groups of EU countries and potential member candidates. The analysis takes into account different pillars of competitiveness, comparing the performance of the core EU 15 countries with later accession countries and potential members. 


\section{Theoretical background}

The competitiveness of a national economy is a concept different from the competitiveness of enterprises. An enterprise that cannot afford to pay its liabilities is bound to leave the market. A country experiencing decreasing competitiveness cannot be eliminated from the market, but the levels of prosperity and living standards in the country will be lowered. That is why assessing national competitiveness requires alternative parameters, such as socio-economic and environmental. According to Faberberg (Faberberg, 1988), national competitiveness represents the country's ability to produce goods and services that meet demands of international markets in free and fair market conditions, maintaining and increasing, at the same time, the real income of the population in the long term. OECD defines competitiveness as the country's ability to produce goods and services that can be exchanged on the world market, in terms of free trade and fair market conditions, while increasing the real income of the population (OECD, 1996). Since the standard of living in a country is determined by productivity that provides the basis for wages growth, strong and stable national currency and increasing returns on capital and hence higher standard of living, the main challenge in terms of national competitiveness is to create conditions for rapid and sustained productivity growth. The main problem of assessing national competitiveness stems from the fact that there is no widely accepted definition of this concept (Šegota et al. 2017) nor the consensus about whether improving competitiveness is the right strategy.

According to Krugman (1994), there is no sense in the meaning of the word competitiveness, when applied to national economy, and it also represents "a dangerous and wrong obsession". He explains that leading countries of the world do not compete with each other and that most economic difficulties do not come from foreign competition, but, on the contrary, they are home-made. However, this "growing obsession with competitiveness" could easily be responsible for trade wars, protectionism and wasting taxpayers' money for enhancing competitiveness. Opposing scholars claim that countries do compete against each other (Dunn, 1994) and that a country's economy is the source of economic means they need, in order to achieve political interests, as well as a source of attractiveness as a location for international business, the strength of national currency, and finally- economic and political power and influence.

This debate is of major importance for measuring competitiveness. Countries are bound to compare themselves in achieving the mentioned goals. The indicators used need to correspond to the underlying economic features. It is very difficult to measure competitiveness using only one indicator, since it cannot be determined by one isolated factor. Instead, there are many factors that affect macroeconomic performance - productivity, innovation, political stability, education, etc. This is the main reason for constructing composite indices, as complex measures that include several groups of indicators. The main advantage of such indicators is that they can 
summarize multi-dimensional process into a single number, which enables comparison between countries and measuring progress over time. However, summarizing complex and often elusive processes into a single measure used for benchmarking countries' performance, if poorly constructed, can be misleading (OECD, 2008). Also, there are many moments in the process of constructing, when indices where subjective judgments are in place, the results may be misused, or subject to political interpretations.

The best known indicator of macro-competitiveness is Global Competitiveness Index (hereafter GCI), which is calculated and published annually by the World Economic Forum and the International Institute of Management Development, starting from 1995. Beside acknowledged analytical, methodological and quantitative weaknesses (Đogo, Stanišić, 2016), it is the most widely used index of competitiveness. In accordance with the requirements contained in the GCI, the term competitiveness itself is defined "as the set of institutions, policies, and factors that determine the level of productivity of a country" (Schwab, 2012).This index is the basis for the international ranking of countries, in terms of business climate. It is of composite nature and involves a number of factors. The underlying assumption is that competitiveness is a complex phenomenon, influenced by a multitude of factors. The above mentioned factors are grouped into 12 pillars of competitiveness, which are organized into three groups. The first group is marked as basic requirements. This group includes the following pillars: (1) institutions, (2) infrastructure, (3) macroeconomic stability, and (4) health and primary education. The second group is called the efficiency enhancers. This group consists of the following pillars: (5) higher education and training, (6) goods market efficiency, (7) labour market efficiency, (8) financial market sophistication, (9) technological readiness and (10) market size. The third group consists of, the so-called, innovation and sophistication factors: business sophistication (11) and innovation (12). The importance of particular groups of pillars depends on the country development level (measured by gross domestic product per capita). For the least developed countries, the first group of pillars is of the utmost importance (basic requirements). For middle-income countries, in addition to basic requirements, efficiency enhancers have a great significance. For developed countries, efficiency enhancers, innovation and sophistication factors are the most important.

\section{Objectives and methodology}

Competitiveness is one of the most commonly used concepts in contemporary economic development policies, both in the regional or national policy frameworks and strategies, especially when it comes to growth or convergence. Since the EU accession process for candidate countries, or potential candidates, means achieving a higher level of economic development, institutional development, more developed infrastructure, markets, as well as the fulfilment of a whole set of 
standards relating to education and health care, their progress in meeting EU standards can also be measured through improving scores for the pillars of the Global Competitiveness Index.

The aim of the paper is to compare the competitiveness of candidate or potential candidate countries with the competitiveness of the EU countries. For the purpose of comparative analysis, the EU countries are divided into groups according to the year of accession to the EU, the EU-15 group of the initial member states, then the member states after the enlargement in 2004, as well as the EU members starting from 2007 and 2013. An overview of the countries listed in these groups is given in Table 1 . The total number of countries that is included in comparative analysis is 33 (28 EU members, 4 candidates and one potential candidate). The analysis does not include Turkey, despite the fact that it is one of the accession candidate countries, because its economic performance differs greatly from the Western Balkan countries which are analysed. Also, Kosovo is excluded from the analysis, because there are no data on competitiveness in Global Competitiveness Report.

Table 1. EU countries, accession candidates and potential accession candidates included in the sample

\begin{tabular}{|c|c|c|}
\hline Group & $\mathrm{N}$ & Countries \\
\hline EU 15 & 15 & $\begin{array}{c}\text { Austria, Belgium, Denmark, Finland, France, Germany, } \\
\text { Greece, Ireland, Italy, Luxembourg, Netherlands, Portugal, } \\
\text { Spain, Sweden, United Kingdom }\end{array}$ \\
\hline $\begin{array}{l}\text { EU members since } \\
2004\end{array}$ & 10 & $\begin{array}{c}\text { Cyprus, Czech Republic, Estonia, Hungary, Latvia, } \\
\text { Lithuania, Malta, Poland, Slovakia, Slovenia }\end{array}$ \\
\hline $\begin{array}{l}\text { EU members since } \\
2007\end{array}$ & 2 & Bulgaria, Romania \\
\hline $\begin{array}{l}\text { EU member since } \\
2013\end{array}$ & 1 & Croatia \\
\hline $\begin{array}{l}\text { Candidates/ Potential } \\
\text { candidates }\end{array}$ & 5 & $\begin{array}{c}\text { Albania, Bosnia and Herzegovina, FYR Macedonia, } \\
\text { Montenegro, Serbia }\end{array}$ \\
\hline
\end{tabular}

Source: Authors' preview according to the EU official website

The time period covered by the analysis in the paper includes Global Competitiveness Reports starting from 2007-2008 to the last report published in October 2017 for the 2017-2018 (World Economic Forum competitiveness dataset, Schwab, 2016; Schwab, 2017).

The first part of the analysis focuses on monitoring the changes in the scores of competitiveness pillars, GCI and GDP per capita for all groups of countries. The goal is to determine the segments of competitiveness in which accession candidate countries or potential accession candidates have the most significant improvement, as well as those segments where the improvement has failed.

The second part of the analysis is based on the Global Competitiveness Report 2017-2018 (Schwab, 2017) and identifies the significance of differences in pillars' 
scores between groups of the EU countries and the accession candidate countries, or potential accession candidates. The methods to be applied in order to identify the differences between groups of countries in the European Union and accession candidates or potential accession candidates, considering competitiveness based on GCI pillars are One-way ANOVA and Post Hoc Test.

\section{Results and discussion}

The first part of the results relates to the analysis of the changes in the competitiveness pillars, which are an integral part of the Global Competitiveness Index. The changes are determined by following the annual percentage changes in the mean values for each group of the countries, and then calculating the overall, cumulative change in the scores of all pillars of competitiveness. The analysis excludes Bosnia and Herzegovina for Global Competitiveness Report 2014-2015, as well as FYR Macedonia for Global Competitiveness Report 2017-2018, due to the lack of data for these countries in indicated years. The results for cumulative changes (in\%) for the period from Global Competitiveness Report 2007-2008 to Global Competitiveness Report 2017-2018 are presented in Table 2. Besides cumulative changes for the period, annual changes could be found in Appendix Table A1.

Table 2. Cumulative changes (in \%) in pillars' scores for the period from Global Competitiven ess Report 2007-2008 to Global Competitiveness Report 2017-2018

\begin{tabular}{|c|c|c|c|c|c|c|c|c|c|c|c|c|}
\hline $\begin{array}{l}\text { Grou } \\
\mathrm{p} \text { of } \\
\text { count } \\
\text { ries }\end{array}$ & $\begin{array}{c}\text { 1st } \\
\text { pillar }\end{array}$ & $\begin{array}{l}\text { 2nd } \\
\text { pillar }\end{array}$ & $\begin{array}{l}\text { 3rd } \\
\text { pillar }\end{array}$ & $\begin{array}{l}\text { 4th } \\
\text { pillar }\end{array}$ & $\begin{array}{l}5 \text { th } \\
\text { pillar }\end{array}$ & $\begin{array}{l}\text { 6th } \\
\text { pillar }\end{array}$ & $\begin{array}{c}7 \text { th } \\
\text { pillar }\end{array}$ & $\begin{array}{c}8 \text { th } \\
\text { pillar }\end{array}$ & $\begin{array}{l}\text { 9th } \\
\text { pillar }\end{array}$ & $\begin{array}{l}\text { 10th } \\
\text { pillar }\end{array}$ & $\begin{array}{l}\text { 11th } \\
\text { pillar }\end{array}$ & $\begin{array}{l}\text { 12th } \\
\text { pillar }\end{array}$ \\
\hline EU15 & -4.43 & 3.43 & -0.31 & 4.10 & 5.05 & -1.60 & 3.98 & -18.14 & 19.93 & 2.23 & -1.00 & 5.61 \\
\hline $\begin{array}{l}\text { EU } \\
2004\end{array}$ & -4.07 & 15.13 & 6.74 & 4.50 & 5.58 & 2.52 & -1.45 & -12.56 & 31.90 & 6.23 & -1.78 & 3.99 \\
\hline $\begin{array}{l}\text { EU } \\
2007 \\
\end{array}$ & 7.87 & 43.84 & 11.91 & 0.86 & 11.06 & 6.75 & -1.90 & -3.16 & 54.93 & 8.21 & -3.60 & 5.75 \\
\hline $\begin{array}{l}\text { EU } \\
2013 \\
\end{array}$ & -10.59 & 17.74 & 1.08 & 6.01 & 5.42 & -1.46 & -14.09 & -14.49 & 45.50 & 4.99 & -7.72 & -14.35 \\
\hline $\begin{array}{l}\text { Can/ } \\
\text { Pot. } \\
\text { cand. }\end{array}$ & 7.10 & 48.46 & -8.80 & 6.41 & 27.09 & 12.45 & -4.72 & -7.21 & 43.75 & 19.05 & 6.11 & 14.80 \\
\hline Total & -2.08 & 13.43 & 1.81 & 4.55 & 8.44 & 2.15 & 0.54 & -13.98 & 29.05 & 6.28 & -0.11 & 6.40 \\
\hline
\end{tabular}

Source: Authors' calculation

The highest cumulative progress is recorded in the $9^{\text {th }}$ pillar - Technological readiness and it amounts to $29.05 \%$ for all countries in the sample. The emphasis is on the fact that the greatest progress in this regard has been made in the EU member states since 2007 (54.93\%), then in Croatia (45.50\%), which has been a 
member of the EU since 2013, and in the candidate or potential candidate countries for the EU membership (43.75\%).

The second largest increase was determined at the $2^{\text {nd }}$ pillar - Infrastructure. When it comes to Infrastructure, candidate or potential candidate countries for the EU membership have recorded the most significant progress $(48.46 \%$ of increase of average score for the group of countries for the pillar Infrastructure).

Generally speaking, for all groups of countries, a significant decline was noted only at the score for $8^{\text {th }}$ pillar - Financial market development. It is interesting to point out that this decline largely relates to the EU 15 member states $(-18.14 \%)$, Croatia (-14.49\%) and the EU member states since 2004 (-12.56\%). When it comes to the candidate or potential candidate countries for the EU membership, negative tendencies were recorded at the $3^{\text {rd }}$ pillar - Macroeconomic environment. The decline in the average score for the Macroeconomic environment in this group of countries is $8.80 \%$.

Further analysis in this paper is based on data from the latest Global Competitiveness Report 2017-2018. Descriptive statistics of pillars' scores is presented in Table 3. The results clearly indicate that the average score for most of the pillars of competitiveness is the highest for the EU 15 countries. The only exception refers to the $3^{\text {rd }}$ pillar - Macroeconomic environment, according to which the best average score has the EU member states group since 2007, then EU member states since 2004 and EU 15 countries are third ranked.

Regardless of the increase in scores for almost all pillars of competitiveness for the period from Global Competitiveness Report 2007-2008 to Global Competitiveness Report 2017-2018, EU candidate or potential candidate countries continue to significantly lag behind in the average scores regarding the EU 15 countries or EU member countries since 2004. Lagging behind is much lower compared to EU member countries since 2007 and 2013 (Table 3).

Table 3. Descriptive statistics of pillars' scores

\begin{tabular}{|c|c|c|c|c|c|}
\hline Pillar & Group of Countries & $\mathrm{N}$ & Mean & $\begin{array}{c}\text { Std. } \\
\text { Deviation }\end{array}$ & Std. Error \\
\hline \multirow[t]{6}{*}{ 1st pillar: Institutions } & EU15 & 15 & 5.036090632 & .7913281102 & .2043200395 \\
\hline & EU members since 2004 & 10 & 4.061271895 & .4662649598 & .1474459266 \\
\hline & EU members since 2007 & 2 & 3.591985155 & .1564398284 & .1106196635 \\
\hline & EU members since 2013 & 1 & 3.454986139 & . & (2) \\
\hline & $\begin{array}{l}\text { Candidates/Potential } \\
\text { candidates }\end{array}$ & 4 & 3.573294328 & .3885459179 & .1942729590 \\
\hline & Total & 32 & 4.408944131 & .8678371300 & .1534133799 \\
\hline 2nd pillar: & EU15 & 15 & 5.639142106 & .3945184065 & .1018642145 \\
\hline \multirow[t]{5}{*}{ Infrastructure } & EU members since 2004 & 10 & 4.678444198 & .2819304156 & .0891542255 \\
\hline & EU members since 2007 & 2 & 3.941282971 & .1687629827 & .1193334495 \\
\hline & EU members since 2013 & 1 & 4.647233769 &. & . \\
\hline & $\begin{array}{l}\text { Candidates/Potential } \\
\text { candidates }\end{array}$ & 4 & 3.776971316 & .4125292286 & .2062646143 \\
\hline & Total & 32 & 4.969039330 & .7811006017 & .1380803831 \\
\hline
\end{tabular}




\begin{tabular}{|c|c|c|c|c|c|}
\hline \multirow{6}{*}{$\begin{array}{l}\text { 3rd pillar: } \\
\text { Macroeconomic } \\
\text { environment }\end{array}$} & EU15 & 15 & 5.237353115 & .9103674501 & .2350558649 \\
\hline & EU members since 2004 & 10 & 5.468301689 & .5851889847 & .1850530053 \\
\hline & EU members since 2007 & 2 & 5.486445168 & .3349926515 & .2368755755 \\
\hline & EU members since 2013 & 1 & 4.849006226 & 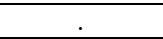 &. \\
\hline & $\begin{array}{l}\text { Candidates/Potential } \\
\text { candidates }\end{array}$ & 4 & 4.432041134 & .4950123175 & .2475061588 \\
\hline & Total & 32 & 5.212292960 & .7811159127 & .1380830897 \\
\hline \multirow{6}{*}{$\begin{array}{l}\text { 4th pillar: Health and } \\
\text { primary education }\end{array}$} & EU15 & 15 & 6.447578630 & .1920916393 & .0495978480 \\
\hline & EU members since 2004 & 10 & 6.237267398 & .2632743435 & .0832546575 \\
\hline & EU members since 2007 & 2 & 5.643674892 & .2229526610 & .1576513385 \\
\hline & EU members since 2013 & 1 & 6.129504810 &. &. \\
\hline & $\begin{array}{l}\text { Candidates/Potential } \\
\text { candidates }\end{array}$ & 4 & 6.034800704 & .1430782916 & .0715391458 \\
\hline & Total & 32 & 6.270075339 & .2984280260 & .0527551202 \\
\hline \multirow{6}{*}{$\begin{array}{l}\text { 5th pillar: Higher } \\
\text { education and } \\
\text { training }\end{array}$} & EU15 & 15 & 5.508104315 & .4512912041 & .1165228879 \\
\hline & EU members since 2004 & 10 & 5.012231401 & .3668024526 & .1159931201 \\
\hline & EU members since 2007 & 2 & 4.513225786 & .1475384041 & .1043254060 \\
\hline & EU members since 2013 & 1 & 4.544574054 & . & . \\
\hline & $\begin{array}{l}\text { Candidates/Potential } \\
\text { candidates }\end{array}$ & 4 & 4.461202318 & .3365284812 & .1682642406 \\
\hline & Total & 32 & 5.129991051 & .5564737476 & .0983715901 \\
\hline \multirow{6}{*}{$\begin{array}{l}\text { 6th pillar: Goods } \\
\text { market efficiency }\end{array}$} & EU15 & 15 & 4.994394757 & .4200208765 & .1084489240 \\
\hline & EU members since 2004 & 10 & 4.656463854 & .2296017128 & .0726064367 \\
\hline & EU members since 2007 & 2 & 4.232302632 & .1261928012 & .0892317855 \\
\hline & EU members since 2013 & 1 & 4.042771213 &. & . \\
\hline & $\begin{array}{l}\text { Candidates/Potential } \\
\text { candidates }\end{array}$ & 4 & 4.112891387 & .3458886869 & .1729443434 \\
\hline & Total & 32 & 4.701234435 & .4713154664 & .0833175906 \\
\hline \multirow{6}{*}{$\begin{array}{l}\text { 7th pillar: Labour } \\
\text { market efficiency }\end{array}$} & EU15 & 15 & 4.635125802 & .5153788833 & .1330702555 \\
\hline & EU members since 2004 & 10 & 4.397268840 & .3060063519 & .0967677050 \\
\hline & EU members since 2007 & 2 & 4.113538180 & .1998032833 & .1412822565 \\
\hline & EU members since 2013 & 1 & 3.766723792 & . & . \\
\hline & $\begin{array}{l}\text { Candidates/Potential } \\
\text { candidates } \\
\end{array}$ & 4 & 3.900092354 & .2889916631 & .1444958315 \\
\hline & Total & 32 & 4.409179531 & .4833777261 & .0854499170 \\
\hline \multirow{6}{*}{$\begin{array}{l}\text { 8th pillar: Financial } \\
\text { market development }\end{array}$} & EU15 & 15 & 4.385930841 & .8638767661 & .2230520219 \\
\hline & EU members since 2004 & 10 & 4.208871827 & .4856789192 & .1535851596 \\
\hline & EU members since 2007 & 2 & 3.942217501 & .2860361057 & .2022580700 \\
\hline & EU members since 2013 & 1 & 3.647207252 &. & . \\
\hline & $\begin{array}{l}\text { Candidates/Potential } \\
\text { candidates }\end{array}$ & 4 & 3.776142370 & .3358800821 & .1679400411 \\
\hline & Total & 32 & 4.203559144 & .6878489733 & .1215956684 \\
\hline \multirow{6}{*}{$\begin{array}{l}\text { 9th pillar: } \\
\text { Technological } \\
\text { readiness }\end{array}$} & EU15 & 15 & 5.917082372 & .4561221359 & .1177702291 \\
\hline & EU members since 2004 & 10 & 5.415466686 & .3405773734 & .1077000220 \\
\hline & EU members since 2007 & 2 & 4.957196613 & .2509439065 & .1774441380 \\
\hline & EU members since 2013 & 1 & 5.038537221 & . & . \\
\hline & $\begin{array}{l}\text { Candidates/Potential } \\
\text { candidates }\end{array}$ & 4 & 4.349934930 & .3530620406 & .1765310203 \\
\hline & Total & 32 & 5.476986644 & .6513070170 & .1151359021 \\
\hline \multirow{6}{*}{$\begin{array}{l}\text { 10th pillar: Market } \\
\text { size }\end{array}$} & EU15 & 15 & 4.836977613 & .7445825682 & .1922503924 \\
\hline & EU members since 2004 & 10 & 3.703601737 & .7942490039 & .2511635882 \\
\hline & EU members since 2007 & 2 & 4.267802607 & .4861018903 & .3437259430 \\
\hline & EU members since 2013 & 1 & 3.617396716 &. & . \\
\hline & $\begin{array}{l}\text { Candidates/Potential } \\
\text { candidates } \\
\end{array}$ & 4 & 3.033315281 & .5934895742 & .2967447871 \\
\hline & Total & 32 & 4.183654519 & .9683210723 & .1711765992 \\
\hline
\end{tabular}




\begin{tabular}{|c|c|c|c|c|c|}
\hline \multirow{6}{*}{$\begin{array}{l}\text { 11th pillar: Business } \\
\text { sophistication }\end{array}$} & EU15 & 15 & 5.181081626 & .5155065432 & .1331032171 \\
\hline & EU members since 2004 & 10 & 4.248869481 & .2707796453 & .0856280423 \\
\hline & EU members since 2007 & 2 & 3.642883878 & .2489886389 & .1760615550 \\
\hline & EU members since 2013 & 1 & 3.792742867 & . & . \\
\hline & $\begin{array}{l}\text { Candidates/Potential } \\
\text { candidates }\end{array}$ & 4 & 3.628286703 & .1945487645 & .0972743822 \\
\hline & Total & 32 & 4.556143020 & .7416812505 & .1311119604 \\
\hline \multirow{6}{*}{$\begin{array}{l}\text { 12th pillar: } \\
\text { Innovation }\end{array}$} & EU15 & 15 & 4.809628731 & .7422465251 & .1916472287 \\
\hline & EU members since 2004 & 10 & 3.616864161 & .3024196084 & .0956334772 \\
\hline & EU members since 2007 & 2 & 3.200751765 & .1637037426 & .1157560265 \\
\hline & EU members since 2013 & 1 & 2.937460400 &. &. \\
\hline & $\begin{array}{l}\text { Candidates/Potential } \\
\text { candidates }\end{array}$ & 4 & 3.048186095 & .2243647486 & .1121823743 \\
\hline & Total & 32 & 4.057649403 & .9147254112 & .1617021353 \\
\hline
\end{tabular}

Source: Authors' calculation

Finally, a comparative analysis of the EU countries and candidate countries' competitiveness was made by comparing the average data of the group of countries that refer to the Global Competitiveness Index and GDP per capita. Descriptive statistics for those two parameters, according to groups of countries is given in Table 4. The results in Table 4 show that the group of countries that is predominantly the best in both observed categories is the EU 15.

It should also be noted that the homogeneity of the EU 15 group, especially when it comes to GDP per capita, is extremely small, and that the standard deviation within the group is 20,471.424 (US\$). Such huge differences within the EU itself, even in the EU-15 countries, clearly indicate that the same models of reform, growth and development cannot be used in all member states (Vlahinić Lenz, Prša, 2015). The differences within the other observed groups are significantly lower, e.g. for candidate or potential candidate countries for the EU membership, the standard deviation is only $1,131.431$ (US\$).

Table 4. Descriptive statistics of GCI and GDP per capita

\begin{tabular}{|c|c|c|c|c|c|}
\hline Pillar & Group of Countries & $\mathrm{N}$ & Mean & $\begin{array}{c}\text { Std. } \\
\text { Deviation }\end{array}$ & Std. Error \\
\hline GCI - Global & EU15 & 15 & 5.139749420 & .4749910677 & .1226421663 \\
\hline Competitiveness & EU member since 2004 & 10 & 4.528491214 & .1934727264 & .0611814480 \\
\hline \multirow[t]{4}{*}{ Index } & EU member since 2007 & 2 & 4.369877754 & .1319688228 & .0933160495 \\
\hline & EU member since 2013 & 1 & 4.190615648 & & \\
\hline & $\begin{array}{l}\text { Candidates/Potential } \\
\text { candidates }\end{array}$ & 4 & 4.084999275 & .1455342556 & .0727671278 \\
\hline & Total & 32 & 4.739110053 & .5301463314 & .0937175165 \\
\hline GDP per capita & EU15 & 15 & $44,481.9533$ & $20,471.42356$ & 5.285 .69883 \\
\hline \multirow[t]{5}{*}{ (US\$) } & EU member since 2004 & 10 & $17,634.8300$ & $4,442.48497$ & $1,404.83710$ \\
\hline & EU member since 2007 & 2 & $8,416.9500$ & $1,482.73221$ & $1,048.45000$ \\
\hline & EU member since 2013 & 1 & $12,095.5000$ & & \\
\hline & $\begin{array}{l}\text { Candidates/Potential } \\
\text { candidates }\end{array}$ & 4 & $5,129.1250$ & $1,131.43057$ & 565.71528 \\
\hline & Total & 32 & $27,906.9844$ & $21,486.63008$ & $3,798.33546$ \\
\hline
\end{tabular}

Source: Authors' calculation 
The ANOVA results are given in Table 5. It is evident that there are statistically significant differences in almost all pillars of competitiveness, except $3^{\text {rd }}$ pillar - Macroeconomic environment and $8^{\text {th }}$ pillar - Financial market development. Also, there are statistically significant differences in GCI score and GDP per capita (sig. 0.000 for all observed parameters).

Table 5. Results of One-way ANOVA

\begin{tabular}{|c|c|c|c|c|c|c|}
\hline & & Sum of Squares & Df & Mean Square & $\mathrm{F}$ & Sig. \\
\hline \multirow[t]{3}{*}{ 1st pillar: Institutions } & $\begin{array}{l}\text { Between } \\
\text { Groups }\end{array}$ & 12.147 & 4 & 3.037 & 7.320 & .000 \\
\hline & $\begin{array}{l}\text { Within } \\
\text { Groups }\end{array}$ & 11.201 & 27 & .415 & & \\
\hline & Total & 23.347 & 31 & & & \\
\hline \multirow[t]{3}{*}{ 2nd pillar: Infrastructure } & $\begin{array}{l}\text { Between } \\
\text { Groups }\end{array}$ & 15.480 & 4 & 3.870 & 30.434 & .000 \\
\hline & $\begin{array}{l}\text { Within } \\
\text { Groups }\end{array}$ & 3.433 & 27 & .127 & & \\
\hline & Total & 18.914 & 31 & & & \\
\hline \multirow[t]{3}{*}{$\begin{array}{l}\text { 3rd pillar: Macroeconomic } \\
\text { environment }\end{array}$} & $\begin{array}{l}\text { Between } \\
\text { Groups }\end{array}$ & 3.382 & 4 & .846 & 1.470 & .239 \\
\hline & $\begin{array}{l}\text { Within } \\
\text { Groups }\end{array}$ & 15.532 & 27 & .575 & & \\
\hline & Total & 18.914 & 31 & & & \\
\hline \multirow[t]{3}{*}{$\begin{array}{l}\text { 4th pillar: Health and primary } \\
\text { education }\end{array}$} & $\begin{array}{l}\text { Between } \\
\text { Groups }\end{array}$ & 1.509 & 4 & .377 & 8.140 & .000 \\
\hline & $\begin{array}{l}\text { Within } \\
\text { Groups }\end{array}$ & 1.252 & 27 & .046 & & \\
\hline & Total & 2.761 & 31 & & & \\
\hline \multirow[t]{3}{*}{$\begin{array}{l}\text { 5th pillar: Higher education } \\
\text { and training }\end{array}$} & $\begin{array}{l}\text { Between } \\
\text { Groups }\end{array}$ & 5.176 & 4 & 1.294 & 7.898 & .000 \\
\hline & $\begin{array}{l}\text { Within } \\
\text { Groups }\end{array}$ & 4.424 & 27 & .164 & & \\
\hline & Total & 9.600 & 31 & & & \\
\hline \multirow[t]{3}{*}{$\begin{array}{l}\text { 6th pillar: Goods market } \\
\text { efficiency }\end{array}$} & $\begin{array}{l}\text { Between } \\
\text { Groups }\end{array}$ & 3.567 & 4 & .892 & 7.254 & .000 \\
\hline & $\begin{array}{l}\text { Within } \\
\text { Groups } \\
\end{array}$ & 3.319 & 27 & .123 & & \\
\hline & Total & 6.886 & 31 & & & \\
\hline \multirow[t]{3}{*}{$\begin{array}{l}\text { 7th pillar: Labour market } \\
\text { efficiency }\end{array}$} & $\begin{array}{l}\text { Between } \\
\text { Groups }\end{array}$ & 2.391 & 4 & .598 & 3.327 & .024 \\
\hline & $\begin{array}{l}\text { Within } \\
\text { Groups }\end{array}$ & 4.852 & 27 & .180 & & \\
\hline & Total & 7.243 & 31 & & & \\
\hline \multirow[t]{3}{*}{$\begin{array}{l}\text { 8th pillar: Financial market } \\
\text { development }\end{array}$} & $\begin{array}{l}\text { Between } \\
\text { Groups }\end{array}$ & 1.676 & 4 & .419 & .871 & .494 \\
\hline & $\begin{array}{l}\text { Within } \\
\text { Groups } \\
\end{array}$ & 12.991 & 27 & .481 & & \\
\hline & Total & 14.667 & 31 & & & \\
\hline $\begin{array}{l}\text { 9th pillar: Technological } \\
\text { readiness }\end{array}$ & $\begin{array}{l}\text { Between } \\
\text { Groups }\end{array}$ & 8.757 & 4 & 2.189 & 13.453 & .000 \\
\hline
\end{tabular}




\begin{tabular}{|c|c|c|c|c|c|}
\hline & $\begin{array}{l}\text { Within } \\
\text { Groups }\end{array}$ & 4.394 & 27 & .163 & \\
\hline & Total & 13.150 & 31 & & \\
\hline \multirow[t]{3}{*}{ 10th pillar: Market size } & $\begin{array}{l}\text { Between } \\
\text { Groups }\end{array}$ & 14.335 & 4 & 3.584 & $6.568 \quad .001$ \\
\hline & $\begin{array}{l}\text { Within } \\
\text { Groups }\end{array}$ & 14.732 & 27 & .546 & \\
\hline & Total & 29.067 & 31 & & \\
\hline \multirow[t]{3}{*}{$\begin{array}{l}\text { 11th pillar: Business } \\
\text { sophistication }\end{array}$} & $\begin{array}{l}\text { Between } \\
\text { Groups }\end{array}$ & 12.497 & 4 & 3.124 & $18.515 \quad \mathbf{. 0 0 0}$ \\
\hline & $\begin{array}{l}\text { Within } \\
\text { Groups }\end{array}$ & 4.556 & 27 & .169 & \\
\hline & Total & 17.053 & 31 & & \\
\hline \multirow[t]{3}{*}{ 12th pillar: Innovation } & $\begin{array}{l}\text { Between } \\
\text { Groups } \\
\end{array}$ & 17.224 & 4 & 4.306 & $13.342 \quad .000$ \\
\hline & $\begin{array}{l}\text { Within } \\
\text { Groups } \\
\end{array}$ & 8.714 & 27 & .323 & \\
\hline & Total & 25.938 & 31 & & \\
\hline \multirow[t]{3}{*}{$\begin{array}{l}\text { GCI - Global Competitiveness } \\
\text { Index }\end{array}$} & $\begin{array}{l}\text { Between } \\
\text { Groups } \\
\end{array}$ & 5.136 & 4 & 1.284 & $9.694 \quad .000$ \\
\hline & $\begin{array}{l}\text { Within } \\
\text { Groups } \\
\end{array}$ & 3.576 & 27 & .132 & \\
\hline & Total & 8.713 & 31 & & \\
\hline \multirow[t]{3}{*}{ GDP per capita (US\$) } & $\begin{array}{l}\text { Between } \\
\text { Groups }\end{array}$ & 8261164923.431 & 4 & 2065291230.858 & $9.216 \quad .000$ \\
\hline & $\begin{array}{l}\text { Within } \\
\text { Groups } \\
\end{array}$ & 6050768508.311 & 27 & 224102537.345 & \\
\hline & Total & 14311933431.742 & 31 & & \\
\hline
\end{tabular}

Source: Authors' calculation

In order to determine in which group of countries and for which parameters there is a statistically significant difference between the average values of the group, the Post Hoc Test was conducted. Since the EU member group since 2013 consists of one member, this is omitted from the analysis, since it is not possible to conduct the Post Hoc Test with single observation within the group.

Results of Post Hoc Test for all pillars of GCI are presented in Table 6. The results indicate that there is a statistically significant difference between the EU 15 countries and other observed groups in the majority of competitiveness pillars. The pillars in which the statistically significant difference is detected are Institutions, Infrastructure, Health and primary education, Higher education and training, Labour market efficiency, Technological readiness, Business sophistication and Innovation.

However, the differences between the EU member states since 2004, and in particular the EU member states since 2007 and candidate or potential candidate countries on the other hand, are not statistically significant for most pillars. The differences that are statistically significant between EU since 2007 and candidate 
or potential candidate countries exists, only with pillars such as Infrastructure and Health and primary education, emphasizing that for Health and primary education the difference is in favour of the candidate countries.

Table 6. Post Hoc Test - Multiple Comparisons of pillar scores

\begin{tabular}{|c|c|c|c|c|c|c|c|}
\hline \multicolumn{8}{|l|}{$\overline{\mathrm{LSD}}$} \\
\hline \multirow[b]{2}{*}{$\begin{array}{l}\text { Dependent } \\
\text { Variable }\end{array}$} & \multirow{2}{*}{$\begin{array}{l}\text { (I) } \\
\text { Accession } \\
\text { Status }\end{array}$} & \multirow{2}{*}{$\begin{array}{l}(\mathrm{J}) \\
\text { Accession } \\
\text { Status }\end{array}$} & \multirow{2}{*}{$\begin{array}{c}\text { Mean } \\
\text { Difference (I- } \\
\mathrm{J})\end{array}$} & \multirow[b]{2}{*}{ Std. Error } & \multirow[b]{2}{*}{ Sig. } & \multicolumn{2}{|c|}{ 95\% Confidence Interval } \\
\hline & & & & & & Lower Bound & Upper Bound \\
\hline \multirow{6}{*}{$\begin{array}{l}\text { 1st pillar: } \\
\text { Institutions }\end{array}$} & \multirow[t]{3}{*}{ EU 15} & EU 2004 & $.9748187370^{*}$ & .2629463497 & .001 & .435297393 & 1.51434008 \\
\hline & & EU 2007 & $1.4441054760^{*}$ & .4848491122 & .006 & .449277272 & 2.43893368 \\
\hline & & $\begin{array}{l}\text { Can./poten. } \\
\text { can. }\end{array}$ & $1.4627963033^{*}$ & .3624465303 & .000 & .719117452 & 2.20647516 \\
\hline & \multirow{2}{*}{$\begin{array}{l}\text { EU } \\
\text { members } \\
\text { since } 2004\end{array}$} & EU 2007 & .4692867390 & .4989056205 & .355 & -.554383038 & 1.49295652 \\
\hline & & $\begin{array}{l}\text { Can./poten. } \\
\text { can. }\end{array}$ & .4879775663 & .3810454618 & .211 & -.293863140 & 1.26981827 \\
\hline & $\begin{array}{l}\text { EU } \\
\text { members } \\
\text { since } 2007 \\
\end{array}$ & $\begin{array}{l}\text { Can./poten. } \\
\text { can. }\end{array}$ & .0186908272 & .5577934409 & .974 & -1.12580678 & 1.16318844 \\
\hline \multirow{6}{*}{$\begin{array}{l}\text { 2nd pillar: } \\
\text { Infrastructure }\end{array}$} & \multirow[t]{3}{*}{ EU 15} & EU 2004 & $.9606979083^{*}$ & .1455812466 & .000 & .661989864 & 1.25940595 \\
\hline & & EU 2007 & $1.6978591346^{*}$ & .2684385549 & .000 & 1.147068716 & 2.24864955 \\
\hline & & $\begin{array}{l}\text { Can./poten. } \\
\text { can. }\end{array}$ & $1.8621707904^{*}$ & .2006699000 & .000 & 1.450430166 & 2.27391141 \\
\hline & \multirow{2}{*}{$\begin{array}{l}\text { EU } \\
\text { members } \\
\text { since } 2004\end{array}$} & EU 2007 & $.7371612263^{*}$ & .2762209942 & .013 & .170402561 & 1.30391989 \\
\hline & & $\begin{array}{l}\text { Can./poten. } \\
\text { can. }\end{array}$ & $.9014728820^{*}$ & .2109672691 & .000 & .468603801 & 1.33434196 \\
\hline & $\begin{array}{l}\text { EU } \\
\text { members } \\
\text { since } 2007\end{array}$ & $\begin{array}{l}\text { Can./poten. } \\
\text { can. }\end{array}$ & .1643116557 & .3088244599 & .599 & -.469343795 & .79796711 \\
\hline \multirow{6}{*}{$\begin{array}{l}\text { 3rd pillar: } \\
\text { Macroecon. } \\
\text { environment }\end{array}$} & \multirow[t]{3}{*}{ EU 15} & EU 2004 & -.2309485742 & .3096404876 & .462 & -.866278376 & .40438123 \\
\hline & & EU 2007 & -.2490920536 & .5709488482 & .666 & -1.42058232 & .92239822 \\
\hline & & $\begin{array}{l}\text { Can./poten. } \\
\text { can. }\end{array}$ & .8053119812 & .4268099576 & .070 & -.070429715 & 1.68105368 \\
\hline & \multirow{2}{*}{$\begin{array}{l}\text { EU } \\
\text { members } \\
\text { since 2004 }\end{array}$} & EU 2007 & -.0181434794 & .5875015179 & .976 & -1.22359702 & 1.18731006 \\
\hline & & $\begin{array}{l}\text { Can./poten. } \\
\text { can. }\end{array}$ & $1.0362605554^{*}$ & .4487116961 & .029 & .115580204 & 1.95694091 \\
\hline & $\begin{array}{l}\text { EU } \\
\text { members } \\
\text { since } 2007\end{array}$ & $\begin{array}{l}\text { Can./poten. } \\
\text { can. }\end{array}$ & 1.0544040348 & .6568466654 & .120 & -.293333998 & 2.40214207 \\
\hline \multirow{6}{*}{$\begin{array}{l}\text { 4th pillar: } \\
\text { Health and } \\
\text { primary } \\
\text { education }\end{array}$} & \multirow[t]{3}{*}{ EU 15} & EU 2004 & $.2103112318^{*}$ & .0878948331 & .024 & .029965931 & .390656532 \\
\hline & & EU 2007 & $.8039037385^{*}$ & .1620700642 & .000 & .471363435 & 1.13644405 \\
\hline & & $\begin{array}{l}\text { Can./poten. } \\
\text { can. }\end{array}$ & $.4127779262^{*}$ & .1211546664 & .002 & .164189085 & .661366768 \\
\hline & \multirow{2}{*}{$\begin{array}{l}\text { EU } \\
\text { members } \\
\text { since } 2004 \\
\end{array}$} & EU 2007 & $.5935925067^{*}$ & .1667687202 & .001 & .251411357 & .935773656 \\
\hline & & $\begin{array}{l}\text { Can./poten. } \\
\text { can. }\end{array}$ & .2024666944 & .1273717140 & .124 & -.058878475 & .463811864 \\
\hline & $\begin{array}{l}\text { EU } \\
\text { members } \\
\text { since } 2007 \\
\end{array}$ & $\begin{array}{l}\text { Can./poten. } \\
\text { can. }\end{array}$ & $-.3911258123^{*}$ & .1864530974 & .045 & -.773695967 & -.00855566 \\
\hline \multirow{5}{*}{$\begin{array}{l}\text { 5th pillar: } \\
\text { Higher } \\
\text { education and } \\
\text { training }\end{array}$} & \multirow{3}{*}{ EU 15} & EU 2004 & $.4958729145^{*}$ & .1652478629 & .006 & .156812307 & .834933522 \\
\hline & & EU 2007 & $.9948785292^{*}$ & .3047020037 & .003 & .369681660 & 1.6200754 \\
\hline & & $\begin{array}{l}\text { Can./poten. } \\
\text { can. }\end{array}$ & $1.0469019972^{*}$ & .2277784598 & .000 & .579539202 & 1.51426479 \\
\hline & \multirow{2}{*}{$\begin{array}{l}\text { EU } \\
\text { members } \\
\text { since } 2004\end{array}$} & EU 2007 & .4990056147 & .3135357751 & .123 & -.144316657 & 1.14232789 \\
\hline & & $\begin{array}{l}\text { Can./poten. } \\
\text { can. }\end{array}$ & $.5510290827^{*}$ & .2394669038 & .029 & .059683582 & 1.04237458 \\
\hline
\end{tabular}


Stanković et al. /Economic Themes, 57(4): 415-432

\begin{tabular}{|c|c|c|c|c|c|c|c|}
\hline & $\begin{array}{l}\text { EU } \\
\text { members } \\
\text { since } 2007\end{array}$ & $\begin{array}{l}\text { Can./poten. } \\
\text { can. }\end{array}$ & .0520234680 & .3505436533 & .883 & -.667232697 & .771279633 \\
\hline \multirow{6}{*}{$\begin{array}{l}\text { 6th pillar: } \\
\text { Goods market } \\
\text { efficiency }\end{array}$} & \multirow[t]{3}{*}{ EU 15} & EU 2004 & $.3379309040^{*}$ & .1431381015 & .026 & .044235779 & .631626029 \\
\hline & & EU 2007 & $.7620921260^{*}$ & .2639336180 & .008 & .220545074 & 1.30363918 \\
\hline & & $\begin{array}{l}\text { Can./poten. } \\
\text { can. }\end{array}$ & $.8815033710^{*}$ & .1973022569 & .000 & .476672579 & 1.28633416 \\
\hline & \multirow{2}{*}{$\begin{array}{l}\text { EU } \\
\text { members } \\
\text { since } 2004\end{array}$} & EU 2007 & .4241612220 & .2715854524 & .130 & -.133086097 & .981408541 \\
\hline & & $\begin{array}{l}\text { Can./poten. } \\
\text { can. }\end{array}$ & $.5435724670^{*}$ & .2074268155 & .014 & .117967797 & .969177137 \\
\hline & $\begin{array}{l}\text { EU } \\
\text { members } \\
\text { since } 2007 \\
\end{array}$ & $\begin{array}{l}\text { Can./poten. } \\
\text { can. }\end{array}$ & .1194112450 & .3036417666 & .697 & -.503610198 & .742432688 \\
\hline \multirow{6}{*}{$\begin{array}{l}\text { 7th pillar: } \\
\text { Labour } \\
\text { market } \\
\text { efficiency }\end{array}$} & \multirow[t]{3}{*}{ EU 15} & EU 2004 & .2378569628 & .1730596912 & .181 & -.117232193 & .592946118 \\
\hline & & EU 2007 & .5215876228 & .3191063034 & .114 & -.133164428 & 1.17633967 \\
\hline & & $\begin{array}{l}\text { Can./poten. } \\
\text { can. }\end{array}$ & $.7350334486^{*}$ & .2385463221 & .005 & .245576825 & 1.22449007 \\
\hline & \multirow[t]{2}{*}{ EU 2004} & EU 2007 & .2837306600 & .3283576772 & .395 & -.390003642 & .957464962 \\
\hline & & $\begin{array}{l}\text { Can./poten. } \\
\text { can. }\end{array}$ & .4971764858 & .2507873185 & .058 & -.017396587 & 1.01174955 \\
\hline & EU 2007 & $\begin{array}{l}\text { Can./poten. } \\
\text { can. }\end{array}$ & .2134458258 & .3671150436 & .566 & -.539812024 & .966703675 \\
\hline \multirow{6}{*}{$\begin{array}{l}\text { 8th pillar: } \\
\text { Financial } \\
\text { market } \\
\text { development }\end{array}$} & \multirow[t]{3}{*}{ EU 15} & EU 2004 & .1770590142 & .2831827704 & .537 & -.403984036 & .758102064 \\
\hline & & EU 2007 & .4437133403 & .5221632282 & .403 & -.627677106 & 1.51510379 \\
\hline & & $\begin{array}{l}\text { Can./poten. } \\
\text { can. }\end{array}$ & .6097884715 & .3903405113 & .130 & -.191124101 & 1.41070104 \\
\hline & \multirow[t]{2}{*}{ EU 2004} & EU 2007 & .2666543261 & .5373015291 & .624 & -.835797348 & 1.3691060 \\
\hline & & $\begin{array}{l}\text { Can./poten. } \\
\text { can. }\end{array}$ & .4327294574 & .4103708214 & .301 & -.409281917 & 1.27474083 \\
\hline & EU 2007 & $\begin{array}{l}\text { Can./poten. } \\
\text { can. }\end{array}$ & .1660751312 & .6007213718 & .784 & -1.06650331 & 1.39865357 \\
\hline \multirow{6}{*}{$\begin{array}{l}\text { 9th pillar: } \\
\text { Technological } \\
\text { readiness }\end{array}$} & \multirow[t]{3}{*}{ EU 15} & EU 2004 & $.5016156861^{*}$ & .1646832242 & .005 & .163713621 & .839517751 \\
\hline & & EU 2007 & $.9598857593^{*}$ & .3036608614 & .004 & .336825137 & 1.58294638 \\
\hline & & $\begin{array}{l}\text { Can./poten. } \\
\text { can. }\end{array}$ & $1.5671474425^{*}$ & .2270001591 & .000 & 1.101381589 & 2.0329133 \\
\hline & \multirow[t]{2}{*}{$\overline{\text { EU } 2004}$} & EU 2007 & .4582700732 & .3124644486 & .154 & -.182854018 & 1.09939416 \\
\hline & & $\begin{array}{l}\text { Can./poten. } \\
\text { can. }\end{array}$ & $1.0655317564^{*}$ & .2386486646 & .000 & .575865144 & 1.55519837 \\
\hline & EU 2007 & $\begin{array}{l}\text { Can./poten. } \\
\text { can. }\end{array}$ & .6072616832 & .3493458738 & .094 & -.109536841 & 1.32406021 \\
\hline \multirow{6}{*}{$\begin{array}{l}\text { 10th pillar: } \\
\text { Market size }\end{array}$} & \multirow[t]{3}{*}{ EU 15} & EU 2004 & $1.1333758761^{*}$ & .3015608860 & .001 & .514624048 & 1.752127704 \\
\hline & & EU 2007 & .5691750061 & .5560507989 & .315 & -.571746992 & 1.710097004 \\
\hline & & $\begin{array}{l}\text { Can./poten. } \\
\text { can. }\end{array}$ & $1.8036623323^{*}$ & .4156729954 & .000 & .950771796 & 2.656552869 \\
\hline & \multirow[t]{2}{*}{$\overline{\text { EU } 2004}$} & EU 2007 & -.5642008700 & .5721715517 & .333 & -1.738199920 & .609798180 \\
\hline & & $\begin{array}{l}\text { Can./poten. } \\
\text { can. }\end{array}$ & .6702864563 & .4370032410 & .137 & -.226370130 & 1.566943042 \\
\hline & $\overline{\text { EU } 2007}$ & $\begin{array}{l}\text { Can./poten. } \\
\text { can. }\end{array}$ & 1.2344873263 & .6397072422 & .064 & -.078083515 & 2.547058167 \\
\hline \multirow{6}{*}{$\begin{array}{l}\text { 11th pillar: } \\
\text { Business } \\
\text { sophistication }\end{array}$} & \multirow[t]{3}{*}{ EU 15} & EU 2004 & $.9322121458^{*}$ & .1676985740 & .000 & .588123094 & 1.276301198 \\
\hline & & EU 2007 & $1.5381977483^{*}$ & .3092208917 & .000 & .903728886 & 2.172666610 \\
\hline & & $\begin{array}{l}\text { Can./poten. } \\
\text { can. }\end{array}$ & $1.5527949230^{*}$ & .2311565320 & .000 & 1.078500897 & 2.027088949 \\
\hline & \multirow[t]{2}{*}{ EU 2004} & EU 2007 & .6059856025 & .3181856726 & .068 & -.046877470 & 1.258848675 \\
\hline & & $\begin{array}{l}\text { Can./poten. } \\
\text { can. }\end{array}$ & $.6205827773^{*}$ & .2430183216 & .017 & .121950369 & 1.119215186 \\
\hline & EU 2007 & $\begin{array}{l}\text { Can./poten. } \\
\text { can. }\end{array}$ & .0145971748 & .3557423967 & .968 & -.715325931 & .744520280 \\
\hline
\end{tabular}




\begin{tabular}{|c|c|c|c|c|c|c|c|}
\hline \multirow[t]{5}{*}{$\begin{array}{l}\text { 12th pillar: } \\
\text { Innovation }\end{array}$} & \multirow[t]{2}{*}{ EU 15} & $\begin{array}{l}\text { EU } 2004 \\
\text { EU } 2007\end{array}$ & $\begin{array}{l}1.1927645696^{*} \\
1.6088769656^{*}\end{array}$ & $\frac{.2319263768}{.4276511084}$ & $\begin{array}{l}.000 \\
.001\end{array}$ & $\frac{.716890952}{.731409371}$ & $\frac{1.668638187}{2.486344560}$ \\
\hline & & $\begin{array}{l}\text { Can./poten. } \\
\text { can. }\end{array}$ & $1.7614426358^{*}$ & .3196884485 & .000 & 1.105496121 & 2.417389150 \\
\hline & \multirow[t]{2}{*}{$\overline{\text { EU } 2004}$} & EU 2007 & .4161123960 & .4400493601 & .353 & -.486794310 & 1.319019102 \\
\hline & & $\begin{array}{l}\text { Can./poten. } \\
\text { can. }\end{array}$ & .5686780663 & .3360932504 & .102 & -.120928321 & 1.258284454 \\
\hline & EU 2007 & $\begin{array}{l}\text { Can./poten. } \\
\text { can. }\end{array}$ & .1525656703 & 4919901413 & .759 & -.856914716 & 1.162046056 \\
\hline
\end{tabular}

Source: Authors' calculation

Analysis of the differences in GCI and GDP per capita points out that the EU 15 countries are statistically and significantly better in these two parameters compared to other observed groups. Also, the difference in GCI between the EU 15 and the EU since 2004 is not statistically significant, nor is the difference between the EU 2007 and candidate countries, or potential candidates for the EU membership. Similar results, for the EU 28 countries in the period 1995 - 2012, can be found in analysis conducted by Simionescu (2015) which shows significant differences between foundation members and CEE economies at national level, when it comes to GDP per capita convergence.

Table7. Post Hoc Test - Multiple Comparisons of GCI and GDP per capita

\begin{tabular}{|c|c|c|c|c|c|c|c|}
\hline \multicolumn{8}{|l|}{ LSD } \\
\hline \multirow{3}{*}{$\begin{array}{l}\text { Dependent } \\
\text { Variable }\end{array}$} & \multirow{3}{*}{$\begin{array}{l}\text { I) } \\
\text { Accession } \\
\text { Status }\end{array}$} & \multirow{3}{*}{$\begin{array}{l}\text { (J) Accession } \\
\text { Status }\end{array}$} & \multirow{3}{*}{$\begin{array}{c}\text { Mean } \\
\text { Difference } \\
(\mathrm{I}-\mathrm{J})\end{array}$} & \multirow[b]{3}{*}{ Std. Error } & \multirow[b]{3}{*}{ Sig. } & \multicolumn{2}{|c|}{ 95\% Confidence Interval } \\
\hline & & & & & & Lower & \\
\hline & & & & & & Bound & Upper Bound \\
\hline \multirow{6}{*}{$\begin{array}{l}\text { GCI - Global } \\
\text { Competitivene } \\
\text { ss Index }\end{array}$} & \multirow[t]{3}{*}{ EU 15} & EU 2004 & $0.611^{*}$ & 0,149 & 0,000 & 0,306 & 0,916 \\
\hline & & EU 2007 & $0.770^{*}$ & 0,274 & 0,009 & 0,208 & 1,332 \\
\hline & & $\begin{array}{l}\text { Can./poten. } \\
\text { can. }\end{array}$ & $1.055^{*}$ & 0,205 & 0,000 & 0,635 & 1,475 \\
\hline & \multirow[t]{2}{*}{ EU 2004} & EU 2007 & 0.159 & 0,282 & 0,578 & $-0,420$ & 0,737 \\
\hline & & $\begin{array}{l}\text { Can./poten. } \\
\text { can. }\end{array}$ & $0.444^{*}$ & 0,215 & 0,049 & 0,002 & 0,885 \\
\hline & $\overline{\text { EU } 2007}$ & $\begin{array}{l}\text { Can./poten. } \\
\text { can. }\end{array}$ & 0.285 & 0,315 & 0,374 & $-0,362$ & 0,932 \\
\hline \multirow{6}{*}{$\begin{array}{l}\text { GDP per } \\
\text { capita (US\$) }\end{array}$} & \multirow[t]{3}{*}{ EU 15} & EU 2004 & $26847.123^{*}$ & 6111,499 & 0,000 & 1430,736 & 39386,884 \\
\hline & & EU 2007 & $36065.003^{*}$ & 11269,048 & 0,003 & 12942,827 & 59187,180 \\
\hline & & $\begin{array}{l}\text { Can./poten. } \\
\text { can. }\end{array}$ & $39352.828^{*}$ & 8424,120 & 0,000 & 22067,961 & 56637,696 \\
\hline & \multirow[t]{2}{*}{$\overline{\text { EU } 2004}$} & EU 2007 & 9217.880 & 11595,755 & 0,434 & $-14574,643$ & 33010,403 \\
\hline & & $\begin{array}{l}\text { Can./poten. } \\
\text { can. }\end{array}$ & 12505.705 & 8856,404 & 0,169 & $-5666,135$ & 30677,545 \\
\hline & EU 2007 & $\begin{array}{l}\text { Can./poten. } \\
\text { can. }\end{array}$ & 3287.825 & 12964,448 & 0,802 & $-23313,024$ & 29888,674 \\
\hline
\end{tabular}

Source: Authors' calculation

With regard to GDP per capita, in addition to the fact that they are significantly lagging behind EU-15 countries, candidate countries or potential candidates for the 
EU membership do not have a statistically significant difference with respect to the EU since 2004 or the EU since 2007 countries. If this fact is added to the previous result, where there are no significant differences or changes when it comes to the scores for pillars of competitiveness, it can be concluded that candidate or potential countries are not notably lagging behind from the new EU member states. However, the differences in macroeconomic environment and parameters that determine the macroeconomic environment exist and these are the results that can be found in research analysis of the EU new member states and SEE regarding FDI, trade and industry performances (Dauti, 2016).

\section{Conclusion}

The presented results clearly indicate that candidate countries, or potential candidate countries for the membership in the European Union, have significantly improved their competitiveness indicators, expressed through the competitiveness pillars of the Global Competitiveness Index. However, the differences are still significant if the EU countries are compared with the countries that are in the accession process.

The scientific contribution of the paper is reflected in the results of the comparative analysis of the EU member states in their original composition (EU 15), then the new EU member states after all three enlargements in the period 2004-2013 and the candidate countries, or potential candidate countries for the EU membership.

The main conclusion is that the differences between the EU 15 and other observed groups are statistically significant in almost all indicators for competitiveness pillars. Also, the EU 15 countries are dominantly better in comparison to other observed groups when it comes to GCI and GDP per capita.

On the other hand, the differences between the EU new member states and the candidate or potential candidate countries for the membership are not so great and, in most cases, not statistically significant, especially if a comparison is being made between the EU member states since 2007 and candidate countries. The negative tendencies in competitiveness of candidate or potential candidate countries for membership in the EU are still poor indicators in the competitiveness pillar of Infrastructure, but also a significant reduction in indicators of Macroeconomic environment pillars. 


\section{References}

Dauti, B. (2016). Trade and foreign direct investment: Evidence from South East European countries and new European Union member states. Proceedings of Rijeka Faculty of Economics: Journal of Economics and Business, 34(1), 63-89.

Dunn, M. H. (1994). Do Nations Compete Economically? A Critical Comment on Prof. Krugman's Essay “Competitiveness: A Dangerous Obsession. Intereconomics, Issue Nov-Dec., 303-308.

Đogo, M. \& Stanišić, N. (2016). Is the Global Competitiveness Report the right measure of macroeconomic competitiveness? Proceedings of Rijeka Faculty of Economics: Journal of Economics and Business, 34 (1), 91-117.

Fagerberg, J. (1988). International competitiveness. The Economic Journal, 98 (391), 355 374.

Krugman P. (1994). Competitiveness. A Dangerous Obsession. Foreign Affairs 73 (3/4), 28 44.

Porter, M. E. (1990). The Competitive Advantage of Nations. New York: Free Press.

Schwab, K. (2012). The Global Competitiveness Report 2012-2013. Geneva: World Economic Forum.

Schwab, K. (2016). The Global Competitiveness Report 2016-2017. Geneva: World Economic Forum.

Schwab, K. (2017). The Global Competitiveness Report 2017-2018. Geneva: World Economic Forum.

Simionescu, M. (2015). About regional convergence clubs in the European Union. Proceedings of Rijeka Faculty of Economics: Journal of Economics and Business, 33(1), 67-80.

Šegota, A., Tomljanović, M., Huđek, I. (2017). Contemporary approaches to measuring competitiveness - the case of EU member states. Proceedings of Rijeka Faculty of Economics: Journal of Economics and Business, 35 (1), 123-150.

OECD (1996). Globalisation and Competitiveness: Relevant Indicators. STI Working papers $1996 / 5$.

OECD (2008) Handbook on Constructing Composite Indicators. Methodology and User Guide. Retrieved from http://www.oecd.org/std/42495745.pdf

Vlahinić Lenz, N, Prša V. (2015). Growth potential of energy sector reforms: new evidence on EU and Southeast European countries by exploring impact on electricity generation. Proceedings of Rijeka Faculty of Economics: Journal of Economics and Business, 33(1), 275-297.

World Economic Forum. Competitiveness Dataset (XLS), Retrieved from http://reports.weforum.org/global-competitiveness-report-2014-2015/methodology/

European Union official website https://europa.eu/european-union/about-eu/countries_en 


\title{
KONKURENTNOST I PROCES PRIDRUŽIVANJA EU: DA LI ZEMLJE KANDIDATI MOGU POSTATI KONKURENTNE KAO ZEMLJE EU?
}

\begin{abstract}
Apstrakt: Ekonomski rast i konkurentnost uobičajeno se analiziraju na nivou nacionalne ekonomije, u tradicionalnim ekonomskim istraživanjima. Problem konkurentnost,i u ovakvom pristupu, uglavnom se posmatra iz perspektive određivanja izvora održivog rasta, osnosno šta ekonomiju čini konkurentnijom od ostalih. Konkurentnost je, dakle, višedimenzionalni koncept koji uključuje niz faktora kao što su institucije, infrastruktura, makroekonomsko okruženje, tržište, ljudski kapital i tehnološki razvoj. Takođe, proces pridruživanja Evropskoj uniji značajno podstiče razvoj određenih kategorija koje su relevantne za ubrzanje ekonomskog razvoja. Cilj rada je proceniti konkurentnost država kandidata, ili potencijalnih kandidata za članstvo u Evropskoj uniji, uporednom analizom njihove konkurentnosti u odnosu na zemlje EU. Rezultati pokazuju da je konkurentnost zemalja EU 15, merena Globalnim indeksom konkurentnosti i BDP-om po stanovniku, statistički značajno veća od konkurentnosti grupe zemalja EU povećane u periodu 2004-2013, takođe u poređenju sa kandidatima za EU ili potencijalnim zemljama kandidatima. Međutim, kada je u pitanju stub konkurentnosti makroekonomskog okruženja, prema poslednjem Izveštaju o globalnoj konkurentnosti (2017-2018), rezultati zemalja EU povezanih sa proširenjima u periodu 2004-2013 statistički su značajno bolji od Zemlje EU 15.
\end{abstract}

Ključne reči: Nacionalna konkurentnost, Evropska unija, proces pridruživanja, zemlje kandidati, statistička analiza.

\section{Authors' biographies}

Jelena J. Stanković, $\mathrm{PhD}$, is an associate professor at the Faculty of Economics, University of Niš for narrow scientific field Mathematics and Statistics in Economics. Her research is focused on quantitative economic analysis, especially methods and models from of operational research and decision theory, as well as statistical analysis in the evaluation of strategies and business decision-making. She has published two monographs, more than 100 papers in scientific and professional journals. She has participated in many research conferences at home and abroad. She is an active member of the Committee for Economic Sciences of the Serbian Academy of Sciences and Arts. Also, she is a head of the several projects realized by the SASA Branch in Nis. Marija Džunić, $\mathrm{PhD}$, is an associate professor at Faculty of Economics, University of Niš, Serbia, Department for General Economic Theory. She is actively engaged as Erasmus+ academic coordinator at the Faculty of Economics, University of Niš. She is the author of numerous articles published in national and ISI indexed international journals. Her research is focused on social capital, social networks and economic growth, with broad research interests aimed at understanding how social factors shape economic outcomes. She is a member of Presidency of the National Scientific Society of Economists.

Vesna Janković Milić, PhD, is an Associate Professor at the Faculty of Economics, University of Nis. She is the author of more than hundred scientific and professional papers. Her research interests include an application of quantitative, especially statistical, methods in economic research. She participates in many project financed by Ministry of Science Republic of Serbia as well as international projects. 


\section{APPENDIX}

Table A1. Annual changes in competitiveness pillars in \% (GCR 2007-2008=100)

\begin{tabular}{|c|c|c|c|c|c|c|c|c|c|c|c|c|c|}
\hline Year & Group & 1st pillar & 2nd & $\begin{array}{l}\text { 3rd } \\
\text { pillar }\end{array}$ & 4th & $\begin{array}{l}\text { 5th } \\
\text { pillar }\end{array}$ & 6th & $\begin{array}{l}\text { 7th } \\
\text { pillar }\end{array}$ & $\begin{array}{c}\text { 8th } \\
\text { pillar }\end{array}$ & $\begin{array}{l}\text { 9th } \\
\text { pillar }\end{array}$ & 10th & 11th & 12th \\
\hline \multirow[t]{6}{*}{$2008-2009$} & EU15 & $-0,11 \%$ & $-0,36 \%$ & $1,30 \%$ & $0,40 \%$ & $-0,86 \%$ & $-1,08 \%$ & $1,20 \%$ & $-2,89 \%$ & $4,46 \%$ & $1,64 \%$ & $-0,31 \%$ & $-1,64 \%$ \\
\hline & EU 2004 & $0,80 \%$ & $0,85 \%$ & $1,07 \%$ & $-0,11 \%$ & $0,33 \%$ & $0,68 \%$ & $0,70 \%$ & $-0,45 \%$ & $7,26 \%$ & $5,72 \%$ & $0,29 \%$ & $-1,11 \%$ \\
\hline & EU 2007 & $3,73 \%$ & $-2,38 \%$ & $2,61 \%$ & $-0,97 \%$ & $3,12 \%$ & $4,61 \%$ & $1,65 \%$ & $5,72 \%$ & $14,85 \%$ & $4,04 \%$ & $0,84 \%$ & $-0,16 \%$ \\
\hline & EU 2013 & $-1,17 \%$ & $0,73 \%$ & $6,36 \%$ & $1,20 \%$ & $0,97 \%$ & $0,25 \%$ & $-0,73 \%$ & $2,41 \%$ & $7,50 \%$ & $3,63 \%$ & $-3,07 \%$ & $-0,61 \%$ \\
\hline & Candidate & $4,47 \%$ & $-0,05 \%$ & $4,56 \%$ & $-1,08 \%$ & $5,24 \%$ & $3,49 \%$ & $4,16 \%$ & $1,42 \%$ & $5,49 \%$ & $8,95 \%$ & $0,46 \%$ & $1,65 \%$ \\
\hline & Total & $0,81 \%$ & $-0,04 \%$ & $1,92 \%$ & $-0,02 \%$ & $0,44 \%$ & $0,33 \%$ & $1,43 \%$ & $-1,04 \%$ & $5,94 \%$ & $3,65 \%$ & $-0,07 \%$ & $-1,04 \%$ \\
\hline \multirow[t]{6}{*}{$2009-2010$} & EU15 & $-2,36 \%$ & $0,18 \%$ & $-5,03 \%$ & $-1,09 \%$ & $-2,02 \%$ & $-3,57 \%$ & $2,60 \%$ & $-11,23 \%$ & $8,19 \%$ & $2,92 \%$ & $-1,79 \%$ & $-2,80 \%$ \\
\hline & EU 2004 & $0,23 \%$ & $5,53 \%$ & $-6,31 \%$ & $-1,72 \%$ & $1,03 \%$ & $-0,06 \%$ & $3,20 \%$ & $-3,96 \%$ & $12,13 \%$ & $5,72 \%$ & $0,06 \%$ & $-0,48 \%$ \\
\hline & EU 2007 & $3,08 \%$ & $1,32 \%$ & $-3,29 \%$ & $-1,34 \%$ & $3,38 \%$ & $4,20 \%$ & $4,87 \%$ & $4,25 \%$ & $18,83 \%$ & $6,87 \%$ & $-1,18 \%$ & $-0,87 \%$ \\
\hline & EU 2013 & $-5,65 \%$ & $8,04 \%$ & $0,74 \%$ & $-1,11 \%$ & $-2,63 \%$ & $-4,48 \%$ & $-6,01 \%$ & $-4,20 \%$ & $21,80 \%$ & $9,15 \%$ & $-8,44 \%$ & $-6,25 \%$ \\
\hline & Candidate & $6,24 \%$ & $8,68 \%$ & $-8,74 \%$ & $-1,93 \%$ & $9,09 \%$ & $5,49 \%$ & $4,51 \%$ & $1,13 \%$ & $16,22 \%$ & $16,12 \%$ & $2,27 \%$ & $4,82 \%$ \\
\hline & Total & $-0,50 \%$ & $2,69 \%$ & $-5,69 \%$ & $-1,41 \%$ & $0,42 \%$ & $-1,05 \%$ & $2,93 \%$ & $-6,53 \%$ & $11,03 \%$ & $5,37 \%$ & $-0,95 \%$ & $-1,36 \%$ \\
\hline \multirow[t]{6}{*}{$2010-2011$} & EU15 & $-3,31 \%$ & $2,04 \%$ & $-6,26 \%$ & $3,05 \%$ & $0,81 \%$ & $-4,94 \%$ & $2,33 \%$ & $-13,98 \%$ & $5,39 \%$ & $1,88 \%$ & $-2,84 \%$ & $-2,69 \%$ \\
\hline & EU 2004 & $-0,32 \%$ & $11,30 \%$ & $-5,43 \%$ & $2,75 \%$ & $4,11 \%$ & $-2,04 \%$ & $2,07 \%$ & $-7,33 \%$ & $8,57 \%$ & $2,51 \%$ & $-3,01 \%$ & $-0,41 \%$ \\
\hline & EU 2007 & $5,62 \%$ & $27,95 \%$ & $-3,15 \%$ & $3,80 \%$ & $5,91 \%$ & $1,96 \%$ & $5,21 \%$ & $-2,24 \%$ & $22,40 \%$ & $3,91 \%$ & $-6,42 \%$ & $-3,25 \%$ \\
\hline & EU 2013 & $-5,55 \%$ & $17,36 \%$ & $0,51 \%$ & $4,06 \%$ & $0,90 \%$ & $-7,91 \%$ & $-11,15 \%$ & $-7,26 \%$ & $22,03 \%$ & $5,20 \%$ & $-13,48 \%$ & $-10,10 \%$ \\
\hline & Candidate & $10,83 \%$ & $36,08 \%$ & $-6,36 \%$ & $2,68 \%$ & $15,20 \%$ & $9,11 \%$ & $6,32 \%$ & $-3,20 \%$ & $18,87 \%$ & $13,37 \%$ & $1,82 \%$ & $8,84 \%$ \\
\hline & Total & $-0,53 \%$ & $9,04 \%$ & $-5,65 \%$ & $2,98 \%$ & $3,70 \%$ & $-2,10 \%$ & $2,57 \%$ & $-9,86 \%$ & $8,97 \%$ & $3,39 \%$ & $-2,83 \%$ & $-1,07 \%$ \\
\hline \multirow[t]{6}{*}{$2011-2012$} & EU15 & $-3,94 \%$ & $3,81 \%$ & $-6,10 \%$ & $2,57 \%$ & $1,47 \%$ & $-5,67 \%$ & $1,82 \%$ & $-14,79 \%$ & $12,21 \%$ & $1,33 \%$ & $-2,48 \%$ & $0,97 \%$ \\
\hline & EU 2004 & $-2,24 \%$ & $11,51 \%$ & $-3,13 \%$ & $2,11 \%$ & $2,79 \%$ & $-1,69 \%$ & $-0,42 \%$ & $-9,43 \%$ & $12,47 \%$ & $2,65 \%$ & $-4,88 \%$ & $-1,11 \%$ \\
\hline & EU 2007 & $2,25 \%$ & $27,51 \%$ & $-1,64 \%$ & $2,96 \%$ & $5,59 \%$ & $1,30 \%$ & $2,40 \%$ & $-2,94 \%$ & $23,04 \%$ & $3,83 \%$ & $-6,92 \%$ & $-3,35 \%$ \\
\hline & EU 2013 & $-7,20 \%$ & $19,81 \%$ & $-0,91 \%$ & $3,11 \%$ & $2,34 \%$ & $-7,16 \%$ & $-11,33 \%$ & $-9,25 \%$ & $30,06 \%$ & $3,74 \%$ & $-11,01 \%$ & $-9,95 \%$ \\
\hline & Candidate & $12,02 \%$ & $44,92 \%$ & $-3,52 \%$ & $1,05 \%$ & $16,78 \%$ & $12,27 \%$ & $5,49 \%$ & $-6,04 \%$ & $23,55 \%$ & $12,53 \%$ & $2,93 \%$ & $9,37 \%$ \\
\hline & Total & $-1,47 \%$ & $10,93 \%$ & $-4,42 \%$ & $2,25 \%$ & $3,83 \%$ & $-2,00 \%$ & $1,28 \%$ & $-11,34 \%$ & $14,45 \%$ & $3,00 \%$ & $-3,01 \%$ & $0,78 \%$ \\
\hline \multirow[t]{6}{*}{$2012-2013$} & EU15 & $-5,43 \%$ & $5,19 \%$ & $-10,03 \%$ & $2,66 \%$ & $2,53 \%$ & $-4,81 \%$ & $2,12 \%$ & $-16,08 \%$ & $14,70 \%$ & $1,63 \%$ & $-2,73 \%$ & $4,22 \%$ \\
\hline & EU 2004 & $-2,84 \%$ & $11,98 \%$ & $-4,64 \%$ & $2,55 \%$ & $3,56 \%$ & $-1,35 \%$ & $-0,38 \%$ & $-10,52 \%$ & $19,39 \%$ & $3,69 \%$ & $-4,87 \%$ & $0,58 \%$ \\
\hline & EU 2007 & $0,91 \%$ & $27,84 \%$ & $4,54 \%$ & $2,12 \%$ & $6,66 \%$ & $1,26 \%$ & $1,96 \%$ & $-2,39 \%$ & $31,13 \%$ & $4,37 \%$ & $-6,18 \%$ & $-2,62 \%$ \\
\hline & EU 2013 & $-8,79 \%$ & $17,84 \%$ & $-1,02 \%$ & $0,56 \%$ & $3,78 \%$ & $-6,08 \%$ & $-8,78 \%$ & $-11,03 \%$ & $26,05 \%$ & $3,55 \%$ & $-10,83 \%$ & $-8,93 \%$ \\
\hline & Candidate & $11,65 \%$ & $44,66 \%$ & $-12,01 \%$ & $0,39 \%$ & $19,24 \%$ & $12,21 \%$ & $1,59 \%$ & $-6,96 \%$ & $29,52 \%$ & $14,02 \%$ & $2,08 \%$ & $10,44 \%$ \\
\hline & Total & $-2,58 \%$ & $11,77 \%$ & $-7,58 \%$ & $2,21 \%$ & $4,97 \%$ & $-1,44 \%$ & $0,94 \%$ & $-12,45 \%$ & $18,71 \%$ & $3,61 \%$ & $-3,19 \%$ & $3,18 \%$ \\
\hline \multirow[t]{6}{*}{ 2013-2014 } & EU15 & $-5,78 \%$ & $4,46 \%$ & $-10,20 \%$ & $3,09 \%$ & $3,57 \%$ & $-5,65 \%$ & $0,91 \%$ & $-18,02 \%$ & $14,27 \%$ & $1,86 \%$ & $-3,20 \%$ & $2,27 \%$ \\
\hline & EU 2004 & $-3,93 \%$ & $11,61 \%$ & $-4,02 \%$ & $2,83 \%$ & $3,98 \%$ & $-1,68 \%$ & $-1,15 \%$ & $-13,41 \%$ & $16,82 \%$ & $4,69 \%$ & $-3,80 \%$ & $0,10 \%$ \\
\hline & EU 2007 & $0,84 \%$ & $32,40 \%$ & $9,70 \%$ & $2,49 \%$ & $6,52 \%$ & $1,91 \%$ & $-0,77 \%$ & $-2,97 \%$ & $34,18 \%$ & $5,32 \%$ & $-4,57 \%$ & $-1,23 \%$ \\
\hline & EU 2013 & $-6,85 \%$ & $18,08 \%$ & $-1,73 \%$ & $0,34 \%$ & $5,17 \%$ & $-4,47 \%$ & $-10,21 \%$ & $-8,64 \%$ & $27,48 \%$ & $4,27 \%$ & $-7,38 \%$ & $-9,00 \%$ \\
\hline & Candidate & $11,45 \%$ & $42,91 \%$ & $-13,55 \%$ & $3,34 \%$ & $21,41 \%$ & $11,83 \%$ & $2,51 \%$ & $-7,50 \%$ & $25,99 \%$ & $15,68 \%$ & $2,84 \%$ & $16,29 \%$ \\
\hline & Total & $-3,05 \%$ & $11,28 \%$ & $-7,41 \%$ & $2,93 \%$ & $5,90 \%$ & $-1,93 \%$ & $0,07 \%$ & $-14,31 \%$ & $17,52 \%$ & $4,25 \%$ & $-2,87 \%$ & $2,67 \%$ \\
\hline \multirow[t]{6}{*}{$2014-2015$} & EU15 & $-6,06 \%$ & $4,62 \%$ & $-8,35 \%$ & $4,13 \%$ & $4,15 \%$ & $-3,69 \%$ & $-0,18 \%$ & $-17,07 \%$ & $16,62 \%$ & $1,40 \%$ & $-3,31 \%$ & $3,27 \%$ \\
\hline & EU 2004 & $-4,22 \%$ & $14,21 \%$ & $-3,74 \%$ & $4,03 \%$ & $6,60 \%$ & $0,98 \%$ & $-2,41 \%$ & $-12,58 \%$ & $19,77 \%$ & $4,59 \%$ & $-2,97 \%$ & $1,83 \%$ \\
\hline & EU2 & $3,25 \%$ & $40,74 \%$ & $8,56 \%$ & $3,05 \%$ & $12,16 \%$ & $7,78 \%$ & $-1,22 \%$ & $1,76 \%$ & $43,99 \%$ & $5,29 \%$ & $-2,43 \%$ & $2,78 \%$ \\
\hline & EU 2013 & $-7,20 \%$ & $19,59 \%$ & $-7,53 \%$ & $2,22 \%$ & $8,35 \%$ & $-1,25 \%$ & $-11,88 \%$ & $-8,35 \%$ & $31,59 \%$ & $3,99 \%$ & $-6,72 \%$ & $-9,56 \%$ \\
\hline & Candidate & $11,04 \%$ & $50,07 \%$ & $-13,99 \%$ & $3,85 \%$ & $26,72 \%$ & $15,61 \%$ & $-1,01 \%$ & $-3,93 \%$ & $32,33 \%$ & $14,68 \%$ & $4,42 \%$ & $15,63 \%$ \\
\hline & Total & $-2,78 \%$ & $13,90 \%$ & $-6,40 \%$ & $4,12 \%$ & $8,40 \%$ & $0,97 \%$ & $-1,21 \%$ & $-12,67 \%$ & $21,69 \%$ & $4,79 \%$ & $-1,78 \%$ & $4,53 \%$ \\
\hline \multirow[t]{6}{*}{$2015-2016$} & EU15 & $-4,70 \%$ & $3,87 \%$ & $-6,44 \%$ & $3,73 \%$ & $4,21 \%$ & $-3,30 \%$ & $1,94 \%$ & $-19,83 \%$ & $18,32 \%$ & $1,67 \%$ & $-2,73 \%$ & $4,78 \%$ \\
\hline & 004 & $-2,76 \%$ & $12,50 \%$ & $2,07 \%$ & $3,99 \%$ & $6,09 \%$ & $1,11 \%$ & $-2,42 \%$ & $-15,10 \%$ & $24,42 \%$ & $5,49 \%$ & $-3,88 \%$ & $3,08 \%$ \\
\hline & EU 2007 & $5,85 \%$ & $38,80 \%$ & $5,87 \%$ & $2,41 \%$ & $11,09 \%$ & $8,87 \%$ & $-0,27 \%$ & $-1,42 \%$ & $48,57 \%$ & $7,51 \%$ & $-2,77 \%$ & $4,84 \%$ \\
\hline & EU 2013 & $-5,97 \%$ & $16,11 \%$ & $-12,72 \%$ & $1,15 \%$ & $7,11 \%$ & $-1,32 \%$ & $-12,57 \%$ & $-15,82 \%$ & $34,26 \%$ & $4,19 \%$ & $-9,04 \%$ & $-8,76 \%$ \\
\hline & Candidate & $8,71 \%$ & $43,56 \%$ & $-11,16 \%$ & $4,70 \%$ & $26,16 \%$ & $13,31 \%$ & $-5,68 \%$ & $-10,76 \%$ & $31,83 \%$ & $17,18 \%$ & $2,86 \%$ & $13,86 \%$ \\
\hline & Total & $-2,20 \%$ & $11,45 \%$ & $-4,00 \%$ & $3,80 \%$ & $7,69 \%$ & $0,71 \%$ & $-1,05 \%$ & $-16,24 \%$ & $23,35 \%$ & $4,64 \%$ & $-2,60 \%$ & $4,90 \%$ \\
\hline \multirow[t]{6}{*}{$2016-2017$} & EU15 & $-3,46 \%$ & $3,64 \%$ & $-4,66 \%$ & $4,09 \%$ & $5,06 \%$ & $-2,07 \%$ & $4,06 \%$ & $-18,71 \%$ & $19,28 \%$ & $0,94 \%$ & $-1,84 \%$ & $5,26 \%$ \\
\hline & EU 2004 & $-3,46 \%$ & $11,82 \%$ & $3,57 \%$ & $4,11 \%$ & $5,37 \%$ & $2,10 \%$ & $-1,96 \%$ & $-14,02 \%$ & $25,15 \%$ & $3,59 \%$ & $-4,50 \%$ & $2,79 \%$ \\
\hline & EU 2007 & $6,95 \%$ & $38,93 \%$ & $9,56 \%$ & $2,03 \%$ & $11,77 \%$ & $8,81 \%$ & $0,62 \%$ & $-3,38 \%$ & $53,92 \%$ & $6,36 \%$ & $-2,86 \%$ & $7,25 \%$ \\
\hline & EU 2013 & $-6,08 \%$ & $16,07 \%$ & $-7,97 \%$ & $0,02 \%$ & $9,03 \%$ & $-0,17 \%$ & $-11,17 \%$ & $-16,14 \%$ & $36,42 \%$ & $2,27 \%$ & $-8,51 \%$ & $-10,76 \%$ \\
\hline & Candidate & $8,22 \%$ & $44,43 \%$ & $-10,00 \%$ & $4,36 \%$ & $25,65 \%$ & $13,63 \%$ & $-5,71 \%$ & $-7,43 \%$ & $35,42 \%$ & $14,15 \%$ & $3,45 \%$ & $14,40 \%$ \\
\hline & Total & $-1,75 \%$ & $11,20 \%$ & $-2,20 \%$ & $3,90 \%$ & $7,93 \%$ & $1,69 \%$ & $0,16 \%$ & $-15,04 \%$ & $24,75 \%$ & $3,31 \%$ & $-2,24 \%$ & $5,20 \%$ \\
\hline \multirow[t]{6}{*}{$2017-2018$} & EU15 & $-4,43 \%$ & $3,43 \%$ & $-0,31 \%$ & $4,10 \%$ & $5,05 \%$ & $-1,60 \%$ & $3,98 \%$ & $-18,14 \%$ & $19,93 \%$ & $2,23 \%$ & $-1,00 \%$ & $5,61 \%$ \\
\hline & & $-4,07 \%$ & $15,13 \%$ & $6,74 \%$ & $4,50 \%$ & $5,58 \%$ & $2,52 \%$ & $-1,45 \%$ & $-12,56 \%$ & $31,90 \%$ & $6,23 \%$ & $-1,78 \%$ & $3,99 \%$ \\
\hline & EU 2007 & $7,87 \%$ & $43,84 \%$ & $11,91 \%$ & $0,86 \%$ & $11,06 \%$ & $6,75 \%$ & $-1,90 \%$ & $-3,16 \%$ & $54,93 \%$ & $8,21 \%$ & $-3,60 \%$ & $5,75 \%$ \\
\hline & EU 2013 & $-10,59 \%$ & $17,74 \%$ & $1,08 \%$ & $6,01 \%$ & $5,42 \%$ & $-1,46 \%$ & $-14,09 \%$ & $-14,49 \%$ & $45,50 \%$ & $4,99 \%$ & $-7,72 \%$ & $-14,35 \%$ \\
\hline & Candidate & $7,10 \%$ & $48,46 \%$ & $-8,80 \%$ & $6,41 \%$ & $27,09 \%$ & $12,45 \%$ & $-4,72 \%$ & $-7,21 \%$ & $43,75 \%$ & $19,05 \%$ & $6,11 \%$ & $14,80 \%$ \\
\hline & Total & $-2,08 \%$ & $13,43 \%$ & $1,81 \%$ & $4,55 \%$ & $8,44 \%$ & $2,15 \%$ & $0,54 \%$ & $-13,98 \%$ & $29,05 \%$ & $6,28 \%$ & $-0,11 \%$ & $6,40 \%$ \\
\hline
\end{tabular}

Source: Authors' calculation based on World Economic forum dataset and GCR 20172016, GCR 2017-2018 